\title{
Design, Development, and Usability Evaluation of a System for Adding and Editing Social Media Banners in the Immersive Street-level 3D Virtual City
}

\author{
Mahmoud Badri ${ }^{1,2)}$, Minna Pakanen ${ }^{1)}$, Paula Alavesa ${ }^{1)}$, Hannu Kukka ${ }^{1)}$, and Timo Ojala ${ }^{1)}$ \\ 1) Center for Ubiquitous Computing \\ Department of Computer Science and Engineering \\ 90014 University of Oulu, Finland \\ fistname.lastname@oulu.fi \\ 2) Rovio Ltd. \\ Keilaranta 7 \\ 02150 Espoo, Finland \\ mahmoud.badri@rovio.com
}

\begin{abstract}
In this paper, we present design and implementation of a system for adding and visualizing social media content in an immersive street-level 3D virtual city environment. The system enables its users to add banners anywhere in the virtual 3D environment, typically on building façades, walls, or on the ground. The banner's owner is then able to edit the banner and select the social media platform to load the content from, thereby creating a social media content banner with a specific ID. The system supports four social media platforms: Facebook, Twitter, Pinterest, and Flickr. Users can also customize banners' position, rotation, and the visual elements such as text, images and colors. To evaluate our system's usability, we conducted an iterative usability evaluation with 12 participants. Each evaluation round with three participants indicated improvements to the system, which were implemented before next evaluation round. Finally, after the last modifications, system was found to be easy to use by average users and only a little help was needed for nonexperienced users. We believe this system could provide added value for the business owners and users by enabling social media content to be add on the 3D virtual city and use it as a marketing platform.
\end{abstract}

Keywords-Social media; $3 D$ city, Collaborative $3 D$ virtual environment.

\section{INTRODUCTION}

Social media is used by businesses for marketing and gaining visibility. Social media platforms such as Facebook and Twitter have evolved over the past few years into having hundreds of millions of active users. Facebook is the most popular social network in the world with 1.71 billion active users per month (end of second quarter of 2016) [1].

Businesses can use social media platforms as additional contact and marketing channels to reach bigger audience, which is proved a worthwhile and profitable strategy. Today most of the businesses are using social media for advertisement, visibility, and branding. It is common that organizations have at least a Facebook page, a YouTube channel, or a Twitter page for each business or even each product category. This helps businesses to reach more people, build trust with its users and improve branding of their organization or products. It also opens a direct channel of communication between the business and its clients; the client can inquire about products or give her/ his feedback and share her/ his experience with the product [2].

Where social platforms such as Facebook are widely used by all age groups, another type of social media, virtual worlds, such as game worlds, are used mainly by younger audiences [3][4]. Virtual worlds are platforms that replicate a three-dimensional environment in which users can appear in the form of personalized avatars and interact with each other as they would in real life. Users of virtual worlds can see the virtual world from the eye of the avatar, in which case it is called "First person view" or they can see their avatar on the screen which they are interacting with it, which is called "Third person view". Virtual worlds are hence social medias in their own. The most prominent example of such virtual worlds is Second Life. Virtual worlds also offer a multitude of opportunities for companies in marketing and advertising. An important progression of digital cities are the $3 \mathrm{D}$ virtual environments built of those cities. A digital city is a virtual representation of the city in which people can interact and share knowledge, experiences, and mutual interests [5]. This concept is gaining more attention from the research community as a promising direction for the future of technology as digital cities are being currently built as virtual environments and $3 \mathrm{D}$ representations of cities, whereas the early digital cities were text based [6]. Some research has been done related to digital cities [5][3][7][8]. The 'digital city'

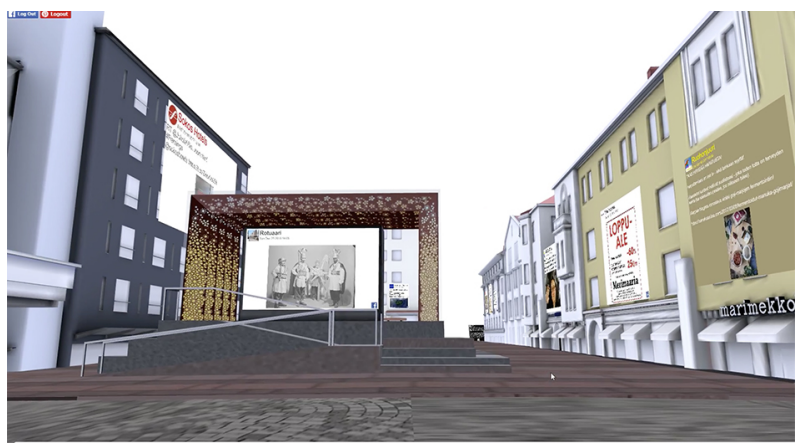

Fig. 1. Social media banners integrated into 3D Virtual City. 
is also considered an integral part of 'smart city' which adds a layer of Internet of Things (IoT) services on top of a digital city and inside virtual cities [5].

Businesses often use plugin to embed their social content on their website or inside another application. Prior research has not investigated how to display businesses' social content inside a virtual 3D environment. Therefore, in this paper we propose a solution for showing social media content within a 3D virtual environment that allows business owners to add their social content on banners placed in a 3D virtual environment, typically on building-walls (Fig. 1). Our system allows businesses to add social content from four social media platforms: Facebook pages, Twitter accounts, Flickr profiles and Pinterest boards. The social media banner shows the recent social media posts as a slide-show. Each slide represents one social media post and includes the post title, message, date and possible images. Also, the profile image of the social page is displayed. The social media icon is displayed at the bottom right of the banner to indicate the source of the social media content on that specific banner.

Next, we will go through related work. After that, we describe our platform for social media integration within 3D city and system components. After that we describe our usability study and report our findings. Finally, we conclude the paper.

\section{RELATED WORK}

\section{A. Social Interaction in 3D Virtual Environments}

Social interaction in a 3D environment plays a role in making the experience more fun and rich. A study conducted by Cole et al. [9], demonstrates that the social interactions in online gaming are a considerable element in the overall enjoyment of playing. Beyond the entertainment and game-play features, virtual worlds are evolving towards a universe in which social and economic interactions are the main drivers [10]. The use of social 3D environment for learning has also been widely studied. Huang et al. [11] carried out a study aiming to identify the factors affecting student learning experience within a social 3D virtual world like Second Life. Garzotto et al. [12] presented FaTe2, an edutainment environment for children that combines collaborative learning and social interaction within 2D and 3D environments. FaTe2 is aimed to be used by children. Siriaraya et al. [13] investigated age differences in the perception of presence in the use of virtual worlds for social interaction. The study concluded that factors such as navigation and prior experience with text messaging tools play a key role in older people's perception of presence in 3D environments. Barkand et al. [14] presented a similar work and findings in the domain of using 3D Virtual Environment for learning and social interaction.

\section{B. Social media for event detection}

Many studies used social media data to predict and/or detect events or to find and/or construct useful information. Stefanidis et al. [15] present a general framework to harvest ambient geospatial information, and analyze it to support situational awareness as it relates to human activities. A more specific study was done by Endarnoto et al. [16] where he used information extraction techniques to get the traffic data from the Twitter account of the police unit in Jakarta, Indonesia. The traffic information was presented on a map view using Google Maps in an android mobile application [16].

Kavanaugh et al. [17] conducted a six-month study aiming to understand the use of social media by government officials, community organizations, businesses and the public. The primary research objectives were to investigate the use and impact of social media and to identify and develop methods to effectively meet a variety of local government and community needs [17]. Diakopoulos et al. [18] presents a visual analytic tool, Vox Civitas, designed to help journalists and media professionals extract news value from large-scale aggregations of social media content around broadcast events. The tool collects, analyzes, aggregates, and visualizes content from major broadcast event [18]. Chae et al. [19] presents a visual analytics approach that provides users with scalable and interactive social media data analysis and visualization including the exploration and examination of abnormal topics and events within various social media data sources, such as Twitter, Flickr, and YouTube.

Kumar et al. [19] presents TwitterTracker, an application designed to track, analyze, and monitor tweets. The purpose of this tool is to help medical first responders gain situational awareness immediately after a disaster or crisis. The system is capable of monitoring and analyzing location and keyword specific tweets with near real-time trending, data reduction, historical review, and integrated data mining tools [20][19]. Similar work has been done by Imran et al. [21] for extracting valuable "information nuggets", brief, self-contained information items relevant to disaster response. Disaster-related datasets consisting of hundreds of thousands of microblogging messages were used in the study. Ratkiewicz et al. [22] created a system for detecting false political campaigns in social media. The system uses a machine-learning framework that combines topological, content-based and crowdsourced features of information diffusion networks on Twitter to detect the early stages of viral spreading of political misinformation [22]. Becker et al.[23], present a query-oriented solution for retrieving social media documents for upcoming events from Twitter, YouTube, and Flickr.

According to our knowledge, no prior study has reported implementation of social media within 3D virtual environment. Only Kukka et al. [24] focused on studying the idea of adding social media content into collaborative virtual 3D city environments in concept level in an anticipated user experience study. Our system is mostly inspired by the public social media integration presented in Kukka et al.[24].

\section{PlatForm FOR VIRTUAL SOCIAL BUSINESS}

The system consists of four main components: social feeds, banners for displaying social feeds, the banner editor for adding, editing and/or deleting the banners, and the database storing banners' data. The application is built using standard HTML5 web technologies. The $3 \mathrm{D}$ virtual environment is rendered using ThreeJS engine [25], which renders the $3 \mathrm{D}$ environment to a WebGL accelerated canvas on a web browser. In the following subsection, we describe the system design and different components of the system. 


\section{A. System Design}

The system architecture is shown in figure 2. The main virtual social business application class lies in the center of the figure. It has two main responsibilities: it glues together all the system components and communicates with the $3 \mathrm{D}$ environment to render the scene after an update happens to the $3 \mathrm{D}$ scene as a result of social media banner updates. The social class is responsible for initializing, cleaning, loading, and authenticating (if necessary) the list of social media platforms. The social media banners are implemented in the banner class and its content is fed by the social class. The editor subsystem is implemented in the banner editor class. This is the subsystem in charge of editing the banners. It is also the communication-handle with the database since only the banner editor is responsible of adding, editing, and deleting banners.

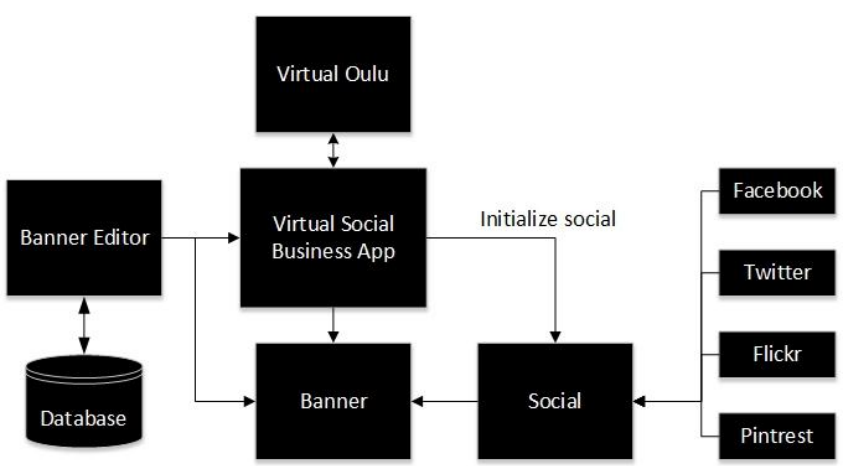

Fig. 2. High level system architecture.

The social feeds are fed to the application via the social class. The application is designed so that adding new social platforms is simple and easy. Adding a new social media platform to the system requires only creating a new social media class and registering it in the social class. No additional modifications are required for the rest of the application.

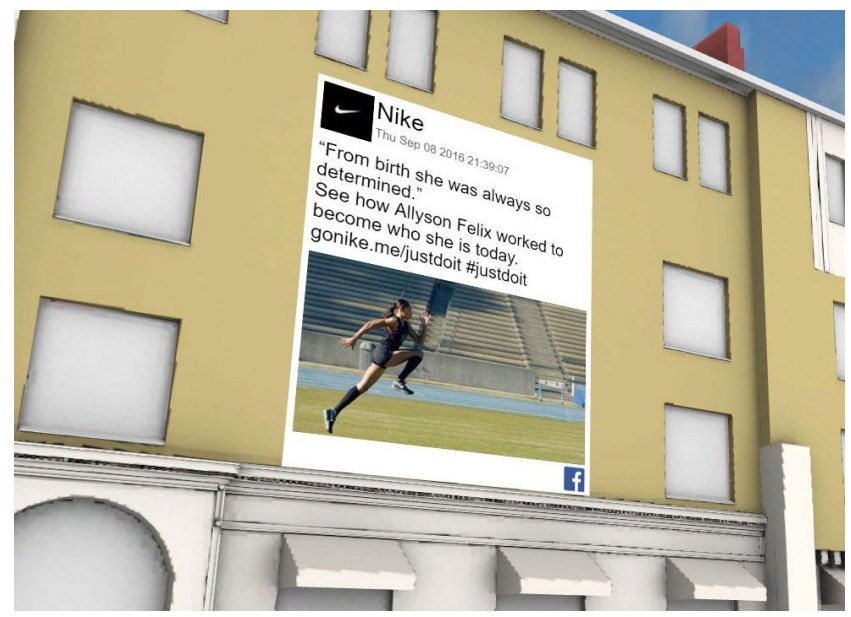

Fig. 3. Social banner displaying post from Nike's Facebook page on a façade of a building.

\section{B. Banners}

Social media feeds i.e. social media contents are displayed on banners positioned on virtual city's façades of the buildings (Fig. 3.). The banner's header includes the social media feed's image, title, and date of the post. Below the header is the content of the banner. The content can be a text, an image, or both. The content of the feed is displayed on the banner one post at a time in a slide-show with about a five second delay. We use smooth animation in the transition between each item of the slide-show on the banner. The delay as well as all properties of the banner can be customized using the banner editor. The social platform icon is displayed on the banners so that the user can easily know the source of each feed.

A banner is a $3 \mathrm{D}$ mesh that is composed of a $2 \mathrm{D}$ plane geometry and a textured material that uses a canvas as a source for the texture. The banner's canvas is used to draw the content of the banner. We used PixiJS engine [26] to facilitate the process of drawing and rendering the content of the banner's canvas. We also used GreenSock animation platform (GSAP) [27] for animating transitions between the banner's pages as well as other animation tasks (such as animating banner's rotation and camera alignment). The banner exposes a well-defined application programming interface (API) for the banner editor to easily manipulate its properties. The banner editor does not edit banners directly, it has to use the banner's API to adjust it.

\section{Banner Editor}

The banner editor is the tool that is used for adding, editing, and deleting banners. Fig. 4 shows the initial version of the banner editor. The editor's panel is divided into different groups, each group controls one aspect of the banners' properties. The banner editor uses the banner API to update the banner properties. Banners are one-sided, which means they are transparent if viewed from the back. In edit mode however, banners' backside is opaque and colored in dark gray. This is useful when adjusting the position and rotation of a specific banner. Below is a description of each group of controls in the banners editor panel and their functionality.

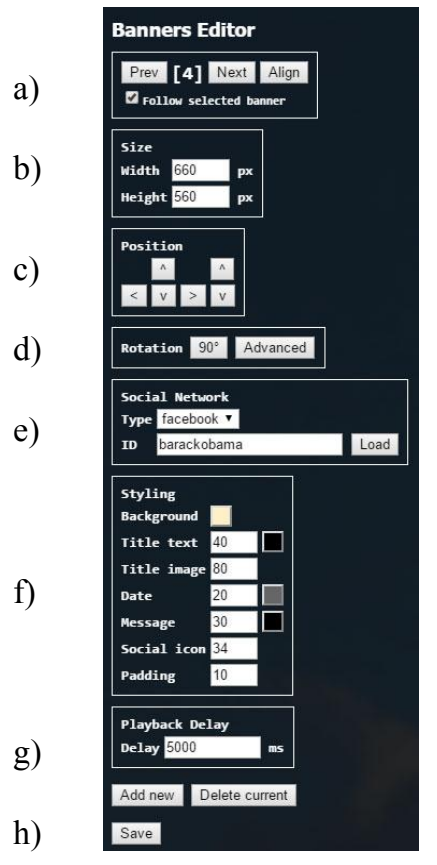

Fig. 4. Banner editor panel. 


\section{Banners Navigation}

The 'Prev/Next' buttons (Fig. 4 a) selects the previous or the next banner to be edited. The number between square brackets is the identifier of the current banner. The 'Align' button aligns the view with the currently selected banner. If the "Follow selected banner" box is checked, the view will automatically align with the current banner when the 'Prev/Next' buttons are clicked. The currently selected banner is highlighted with a red border so that the user can easily recognize it while moving inside the $3 \mathrm{D}$ scene.

\section{E. Size}

The size of the banner (Fig. $4 \mathrm{~b}$ ) can be adjusted in terms of width and height. For example, if the feed contains only short messages without images (like a Twitter feed might) it may be better to have a wider banner with less height. Whereas if the feed posts have both text and images, banner needs to be both wider and higher.

\section{F. Position}

The position of the banner is adjusted by arrows (Fig. $4 \mathrm{c}$ ). Banners can be moved in 6 directions: front, back, left, right, up, and down. Banner movement is relative to the banner angle. Hence, the right button will move the banner to the right of its current facing direction and similarly for the rest of the directions.

\section{G. Rotation}

For basic use, e.g. most of the cases, only the ' $90^{\circ}$ ' button is enough to manipulate the rotation of a specific banner (Fig. $4 \mathrm{~d}$ ). Rotation button turns the banner $90^{\circ}$ at a time around the Y-axis. If a custom rotation or rotations around other axes angle are required, one can access the custom rotation controls panel as show in Fig. 5. From this panel, the user can rotate the banner around any of the $\mathrm{x}, \mathrm{y}$, and $\mathrm{z}$ axes at any angle.

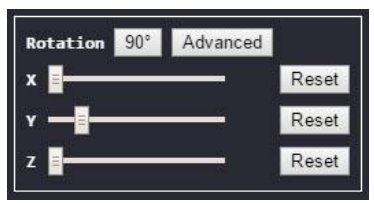

Fig. 5. Advanced rotation controls.

\section{H. Social Networks}

The social network to be shown on a banner, is selected from 'type' drop down list (Fig. 4 e). The list of social networks is populated dynamically by the 'social class'. Currently four social networks are implemented: Facebook, Twitter, Flickr, and Pinterest. Next, the identifier i.e. ID field where the user provides the ID for the social media content to load. The ID is used differently depending on the social platform. In Facebook, the ID represents a page ID. In Twitter and Flickr, it is a user profile ID. In Pinterest, the ID includes the user ID plus a board ID separated by a slash, for example 'Adidas/all-inspiration'. After a correct ID is inserted, clicking the 'Load' button will load the social media feed instantly on the banner-surface.

\section{Styling}

To customize the visual look of the visual elements of the banner, 'Styling' options are used (Fig. 4 e). This includes the banner's background, the header's text size and color, header's image size, date text size and color, body message size and color, social icon size and the padding from the banner's edges. All styling updates are rendered instantly on the banner so the user can see the changes in real time. This makes it intuitive and easy to customize the look of the banner.

\section{J. Playback Delay}

The 'Playback delay' of the social media content of the banner is adjusted in milliseconds (Fig. 4 g). By a default, a five seconds delay is set. The playback delay is the time taken by each slide on the banner before the next slide is displayed. To assure smooth transition of banner slides a fade-in animation is used.

\section{K. Add New / Delete Current}

The 'Add new' button adds a new banner to the scene (Fig. $4 \mathrm{~h}$ ). The system will try first to detect a building-wall in the current user's view. If it fails to do so, the new banner will be placed in front of the user. The new banner will have default values for all the settings and the user can begin customizing the banner settings. The 'Delete current' button will delete the selected banner (Fig. $4 \mathrm{~h}$ ). Any changes done on the banner editor panel will not persist until the 'Save' button is clicked, except for adding and deleting banner which will be executed immediately.

\section{Database}

We use a MySQL database for storing the banners' data. The database has a single table labeled 'banners' for this purpose. The 'banners' table contains a column for each setting in the banner editor panel (Position, Rotation, Social media Id, Size, Playback delay, and Styling). Three Hypertext Preprocessor (PHP) scripts are used to add, update and delete the banners' data. The banner editor is the only entity in the system that communicates with the database.

\section{EVALUATION}

We conducted a usability evaluation for the banner editor panel with three types of users: high, medium, and low experience with $3 \mathrm{D}$ virtual environments. The aim was to find out how easy it is for users to add, edit, and delete banners. The system was updated iteratively after three usability evaluations. This approach allowed us to gather more findings iteratively and improve usability of the system.

\section{A. Participants}

We recruited 12 participants with ages varying from 21 to 32 with a mean of 25. Participants were recruited from the university campus. We chose people who had different experience levels of using 3D virtual environments: four of them were experienced, four of them had basic experience, and the last four had little or no experience of them. Participants took part in the evaluation voluntarily. Sessions were video recorded with the consent from the participants.

\section{B. Evaluation Tasks}

Evaluation sessions began with a brief explanation of the application and its features. After that participants were shown the application in the standard view (without the banner editor). In the same time researcher explained the idea of the social banners. The actual usability evaluation had following ten tasks: 
1. Add a new empty banner on the specified building surface (pointed by the researcher).

2. Use the Move/rotate controls to position the banner to the center of the building's surface.

3. Set the banner to load a feed from Nike's Facebook page (feed id: "Nike").

4. Resize the banner to double its current size.

5. Customize the banner style by changing its background color to yellow and make the font and figure larger.

6. Navigate to the banner number 4 and change its playback delay to 6 seconds.

7. Navigate to banner number 3 and adjust its social media settings to display University of Oulu Twitter feed (id: "UniOulu").

8. Navigate to the banner you just created and delete it.

9. Navigate to banner number 5 and use the advanced rotation feature, to make banner aligned with the building surface.

10. Use the 'Follow selected banner' and 'Align' features and explain what you think they are supposed to do.

The study was conducted in controlled setting. Users interacted with the banner editor and social virtual environment with a laptop and a mouse. The study tasks were printed on a paper and the paper was located beside the laptop. The moderator sat beside the participant to be able to gather observation notes and guide participants if needed. Moderator guided participants in basic navigation of the $3 \mathrm{D}$ scene, especially this application requires clicking the view and drag to navigate around, which was not intuitive to some participants. Also, if participants did not understand what they were supposed to do in the task, such as: on which building façade the banner should be add. In addition, moderator helped to locate banner, it was accidentally inserted behind the façade. Evaluation sessions for experienced 3D users took place before the sessions for participants with less or no prior 3D experience.

Participants were instructed to think aloud while reading and trying to complete the tasks. Also, they were guided to ask for help from the researcher if they did not know what to do. After participants completed given ten tasks they were asked to fill 'system usability scale' (SUS) form [28] that contains 10 standardized usability questions with a five-point scale from strongly disagree (1) to strongly agree (5). The total evaluation session took about 20 minutes. At the end, participants were asked if they have comments based on their experience with the system and if they have additional ideas for improving the system.

\section{ITERATIVE DEVELOPMENT CYCLES}

In this section, we describe the findings in each subevaluation milestone and the development done to the application based on them.

After the first three evaluation sessions, findings revealed some usability issues with the banners editor panel, Fig. 6 shows the updated banners editor panel after the 2 nd evaluation round.
The following updates were done to the application to tackle the most obvious usability issues: we moved the Add/Delete button (Fig. 6 a) to the top of the panel so that the basic tasks of adding and removing banners are obvious for the users. We also split the panel into three main sections with white horizontal lines that were named as follows: 'Add/Remove banners', 'Navigate banners', and 'Edit selected banner'. Participants were mixing the 'Forward/Backward' and the 'Up/Down' buttons because of symbol (arrow up and down) and their positions (Fig. 4 c). Therefore, instead of symbols 'Forward' and 'Backward' texts were used and the positions of the Forward/Backward and 'Up/Down' buttons were swapped (Fig. 6 d).

After the $2^{\text {nd }}$ evaluation cycle we renamed the button that rotates the banner $90^{\circ}$ to 'Rotate $90^{\circ}$ ' (Fig. 6 e). Moreover, we added hints for all the editor controls that appears on roll over. We added also a checkbox to put hints 'On/Off' (Fig. 6 a). The playback delay (Fig. $6 \mathrm{~h}$ ) setting was also updated to show the time in seconds rather than in milliseconds.

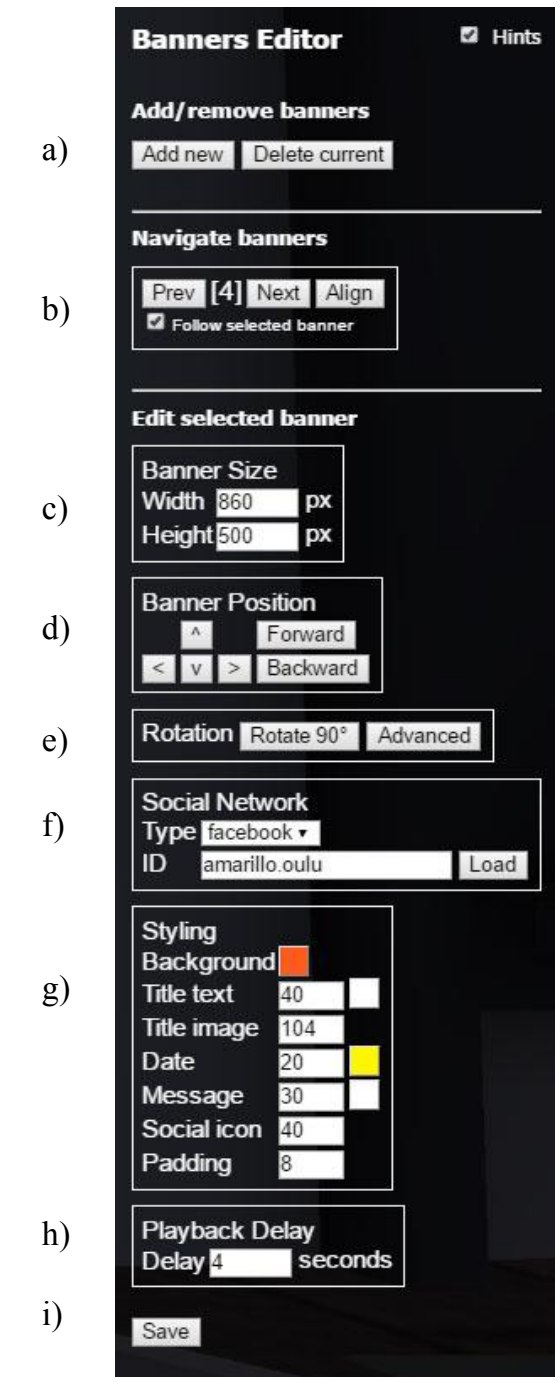

Fig. 6. Iterated banner editor panel.

In the 3rd evaluation round two participants were trying to select banners by clicking on them and they found in addition that navigating between banners using the banner editor panel 
proved to be cumbersome. Therefore, we added a feature that allows the user to select a banner by simply clicking on it in the $3 \mathrm{D}$ scene instead of having to navigate to it by using navigation controls.

The 4th and last evaluation round indicated that nonexperienced users do not know the keyboard and mouse controls for navigating in the $3 \mathrm{D}$ scene. Therefore, we added a splash screen that displays a brief introduction to the controls. The splash screen also shows the name of the application and the loading progress of the 3D models which is important, as models are more than $100 \mathrm{MB}$, which can take a while to upload especially through a slow Internet connection.

\section{RESULTS}

It was apparent that less experienced participants spend more time in understanding the system features and getting accustomed to the system (Fig. $7 \&$ TABLE I. ).

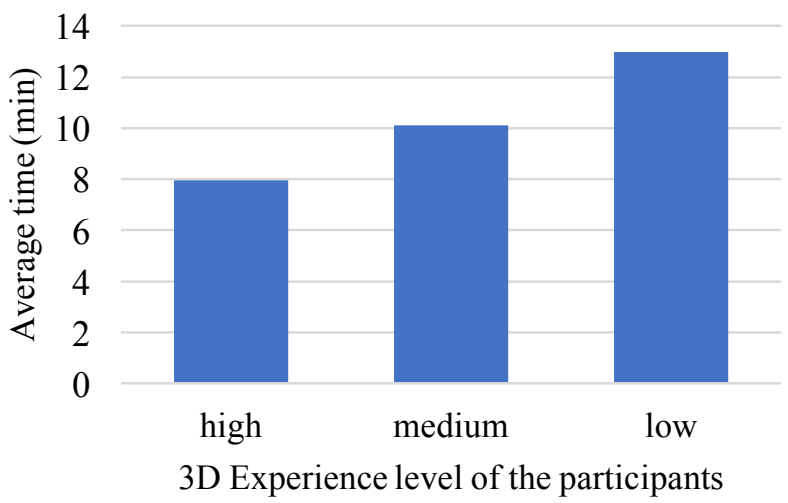

Fig. 7. Average time spent by participants to finish an evaluation session.

SUS scores were counted by following instructions in Brook [28]. TABLE I. presents the results of each participants' SUS form.

TABLE I. INDIVIDUAL AND THE MEAN SUS SCORES FOR EXPEREINCED USERS (HIGH), USERS WITH MEDIUM EXPERIENCE (MEDIUM), AND USERS WITH LOW EXPEREINCE (LOW).

\begin{tabular}{|c|c|c|c|c|}
\hline Participant & $\begin{array}{l}3 \mathrm{D} \\
\text { Experience }\end{array}$ & $\begin{array}{l}\text { Time } \\
(\mathrm{min})\end{array}$ & $\begin{array}{l}\text { SUS } \\
\text { score }\end{array}$ & $\begin{array}{c}\text { Mean SUS } \\
\text { score }\end{array}$ \\
\hline P1 & \multirow[t]{4}{*}{ high } & 8,45 & 90 & \multirow[t]{4}{*}{80,0} \\
\hline $\mathrm{P} 2$ & & 7,50 & 75 & \\
\hline P3 & & 6,50 & 70 & \\
\hline P4 & & 9,40 & 85 & \\
\hline P5 & \multirow[t]{4}{*}{ medium } & 9,37 & 80 & \multirow[t]{4}{*}{86,3} \\
\hline P6 & & 9,50 & 100 & \\
\hline P7 & & 9,78 & 75 & \\
\hline P8 & & 11,75 & 90 & \\
\hline P9 & \multirow[t]{4}{*}{ low } & 15,50 & 80 & \multirow[t]{4}{*}{84,4} \\
\hline $\mathrm{P} 10$ & & 10,50 & 92,5 & \\
\hline $\mathrm{P} 11$ & & 13,30 & 87,5 & \\
\hline $\mathrm{P} 12$ & & 12,60 & 77,5 & \\
\hline
\end{tabular}

The TABLE I shows that the SUS scores for all the three user groups (high 80, medium 86, low 84) are above average, which indicates that our system is highly usable and the learning curve is steep. Even users with no prior skills with $3 \mathrm{D}$ virtual environments can learn to use our system with minimal instructions.

Though the results of the SUS form gave positive indications for system usability, participants' comments, however, revealed some usability issues that need to be addressed. Firstly, all participants did not realize that they need to click the save button after updating a banner. The only case when they clicked the save button was when they were using buttons located near the bottom area of the panel where the 'Save' button is located. Therefore, a pop-up message, such as: "You have unsaved changes" with a 'Save' button (Fig. 5 i) is needed.

Another issue that caused confusion to some participants was that sometimes when a participant added a new banner to a façade of the building, the banner was positioned a little bit behind the wall and thus was not visible. This happened because the buildings' geometry used as a collider is uneven (not flat), hence casting a ray from the camera towards the building-wall may hit a point positioned a little bit behind the wall, which causes the banner to be laid behind of it. To fix this issue, collider geometry (box colliders) suitable for our system are needed.

We also noticed during the evaluation sessions that participants usually do not read the instructions provided in the form of tool tips on the controls. This could be due to the pressure of being subject to a video-recorded evaluation. When the researcher pointed them to read the instructions for unclear features, it apparently helped them to understand the purpose of those features. Understanding what the 'Align' button does was challenging for participants with zero to basic experience. Yet reading the tool tip hint allowed all participants to understand it. On the other hand, the "Follow selected banner" checkbox was hard for all users to understand. Therefore, more comprehensive tool tip text is needed to make sure the feature is as clear as possible.

\section{CONCLUSION}

We implemented an editor system for adding and editing social media content in a $3 \mathrm{D}$ virtual environment in the form of social media banners. The system can be used as an advertisement tool by businesses to display their social media content in a 3D virtual city. It could also be used as a rich source of information, by adding banners that display content about the locations they are placed in. This can be especially handy for instance on cultural heritage sites or tourist attractions. To evaluate the usability of the system, we conducted usability study with 12 participants. SUS form was used and its findings indicated that the system was easy to use and usable. Yet basic instructions are needed for the users with zero to little knowledge with 3D environments.

We believe that realistic looking 3D virtual city environments will become more popular in the near future. Consequently, the importance of integrating social media in those is equally important. As the focus of this study was on usability of the system adding, editing, and deleting public social media content in $3 \mathrm{D}$ virtual city, we were not able to investigate user experiences of the overall idea of adding social media in this context. In addition, we did not take into consideration how users own social media could be integrated and interacted within 
the scene. Also, it would be important to study what kind of impact social media integration have on user's virtual experience. Another important research topic is the ownership of the banners. Currently all the banners are owned by a single user, however, everyone can edit all the banners. In addition, no local business owners, did not take part in our study. Considering the relevance of our system, we should add business owners and the community into our study as well. Before mentioned topics should be addressed in the future research.

\section{A. Limitations}

Although the SUS scores obtained are high and SUS questionnaire is a validated method of assessing system usability our study could benefit from a bigger sample size.

\section{ACKNOWLEDGEMENTS}

This work has been supported by the Open Innovation Platforms spearhead project and the Open City Model as Open Innovation Platform pilot project funded by the ERDF and the City of Oulu under the Six City Strategy program, and the COMBAT project (293389) funded by the Strategic Research Council at the Academy of Finland.

\section{REFERENCES}

[1] Statista, "Number of Facebook users worldwide 2008-2017 | Statistic," Statista. [Online]. Available: https://www.statista.com/statistics/264810/number-of-monthly-activefacebook-users-worldwide/. [Accessed: 07-Dec-2016].

[2] J. Strauss, E-marketing. Routledge, 2016.

[3] N. Yee, "The Demographics, Motivations, and Derived Experiences of Users of Massively Multi-User Online Graphical Environments," Presence: Teleoperators and Virtual Environments, vol. 15, no. 3, pp. 309-329, 2006.

[4] P. Van den Besselaar and D. Beckers, "Demographics and sociographics of the Digital City," in Community Computing and Support Systems, Springer, 1998, pp. 108-124.

[5] T. Ishida, "Digital city kyoto," Communications of the ACM, vol. 45, no. 7, pp. 76-81, 2002.

[6] A. Cocchia, "Smart and digital city: A systematic literature review," in Smart city, Springer, 2014, pp. 13-43.

[7] R. Linturi, M.-R. Koivunen, and J. Sulkanen, "Helsinki Arena 2000Augmenting a real city to a virtual one," in Digital Cities, Springer, 2000, pp. 83-96.

[8] K. Su, J. Li, and H. Fu, "Smart city and the applications," in Electronics, Communications and Control (ICECC), 2011 International Conference on, 2011, pp. 1028-1031.

[9] H. Cole and M. D. Griffiths, "Social interactions in massively multiplayer online role-playing gamers," CyberPsychology \& Behavior, vol. 10, no. 4, pp. 575-583, 2007.

[10] A. Hendaoui, M. Limayem, and C. W. Thompson, "3D social virtual worlds: research issues and challenges," IEEE internet computing, vol. 12, no. 1, 2008.

[11] Y.-C. Huang, S. J. Backman, L.-L. Chang, K. F. Backman, and F. A. McGuire, "Experiencing student learning and tourism training in a $3 \mathrm{D}$ virtual world: an exploratory study," Journal of Hospitality, Leisure, Sport \& Tourism Education, vol. 13, pp. 190-201, 2013.

[12] F. Garzotto and M. Forfori, "Hyperstories and social interaction in 2D and 3D edutainment spaces for children," in Proceedings of the seventeenth conference on Hypertext and hypermedia, 2006, pp. 57-68.

[13] P. Siriaraya and C. S. Ang, "Age differences in the perception of social presence in the use of $3 \mathrm{D}$ virtual world for social interaction," Interacting with Computers, vol. 24, no. 4, pp. 280-291, 2012.

[14] J. Barkand and J. Kush, "GEARS a 3D Virtual Learning Environment and Virtual Social and Educational World Used in Online Secondary Schools.," Electronic Journal of e-learning, vol. 7, no. 3, pp. 215-224, 2009.

[15] A. Stefanidis, A. Crooks, and J. Radzikowski, "Harvesting ambient geospatial information from social media feeds," GeoJournal, vol. 78, no. 2, pp. 319-338, 2013.

[16] S. K. Endarnoto, S. Pradipta, A. S. Nugroho, and J. Purnama, "Traffic condition information extraction \& visualization from social media twitter for android mobile application," in Electrical Engineering and Informatics (ICEEI), 2011 International Conference on, 2011, pp. 1-4.

[17] A. L. Kavanaugh et al., "Social media use by government: From the routine to the critical," Government Information Quarterly, vol. 29, no. 4, pp. 480-491, 2012.

[18] N. Diakopoulos, M. Naaman, and F. Kivran-Swaine, "Diamonds in the rough: Social media visual analytics for journalistic inquiry," in Visual Analytics Science and Technology (VAST), 2010 IEEE Symposium on, 2010, pp. 115-122.

[19] J. Chae et al., "Spatiotemporal social media analytics for abnormal event detection and examination using seasonal-trend decomposition," in Visual Analytics Science and Technology (VAST), 2012 IEEE Conference on, 2012, pp. 143-152.

[20] S. Kumar, G. Barbier, M. A. Abbasi, and H. Liu, "TweetTracker: An Analysis Tool for Humanitarian and Disaster Relief," in In ICWSM, 2011.

[21] M. Imran, S. M. Elbassuoni, C. Castillo, F. Diaz, and P. Meier, "Extracting information nuggets from disaster-related messages in social media," Proc. of ISCRAM, Baden-Baden, Germany, 2013.

[22] J. Ratkiewicz, M. Conover, M. R. Meiss, B. Gonçalves, A. Flammini, and F. Menczer, "Detecting and Tracking Political Abuse in Social Media.," ICWSM, vol. 11, pp. 297-304, 2011.

[23] H. Becker, D. Iter, M. Naaman, and L. Gravano, "Identifying content for planned events across social media sites," in Proceedings of the fifth ACM international conference on Web search and data mining, 2012, pp. 533542.

[24] H. Kukka, M. Pakanen, M. Badri, and T. Ojala, "Immersive Street-level Social Media in the 3D Virtual City: Anticipated User Experience and Conceptual Development," in Proceedings of the 2017 ACM Conference on Computer Supported Cooperative Work and Social Computing, 2017, pp. 2422-2435.

[25] R. Cabello, "three.js - Javascript 3D library." [Online]. Available: https://threejs.org/. [Accessed: 07-Dec-2016].

[26] “PixiJS v4." [Online]. Available: http://www.pixijs.com/. [Accessed: 07Dec-2016].

[27] "GSAP, the standard for JavaScript HTML5 animation | GreenSock." [Online]. Available: https://greensock.com/gsap. [Accessed: 07-Dec2016].

[28] J. Brooke, "SUS-A quick and dirty usability scale," Usability evaluation in industry, vol. 189, no. 194, pp. 4-7, 1996. 